\title{
Derivation of the Coordinate and the Stress Components Transformation Equation in Wellbore Stability Mechanics Analysis
}

\author{
Yang Zaisheng \\ ${ }^{1 .}$ Low-permeability Oil and Gas Exploration and \\ Development of National Engineering Laboratory, Changqing \\ Oilfield Company,Xi'an, 710021, China \\ 2. State Key Lab. of Oil \& Gas Reservoir Geology and \\ Exploitation, SouthWest Petroleum University \\ Chendu, 610500, China \\ yzs_cq@petrochina.com.cn
}

\author{
Ma Tianshou \\ State Key Lab. of Oil \& Gas Reservoir Geology and \\ Exploitation, SouthWest Petroleum University \\ Chendu, 610500, China \\ matianshou@126.com
}

\author{
Chen Ping \\ State Key Lab. of Oil \& Gas Reservoir \\ Geology and Exploitation, SouthWest \\ Petroleum University \\ Chendu, 610500, China \\ Chenping@swpu.edu.cn
}

\author{
OuYang Yong \\ Low-permeability Oil and Gas \\ Exploration and Development of \\ National Engineering Laboratory, \\ Changqing Oilfield Company \\ Xi'an, 710021, China \\ oyy_cq@petrochina.com.cn
}

\author{
Wu Xuesheng \\ Low-permeability Oil and Gas \\ Exploration and Development of \\ National Engineering Laboratory, \\ Changqing Oilfield Company \\ Xi'an, 710021, China \\ wxs_cq@petrochina.com.cn
}

\begin{abstract}
Based on CauChy formula and the basic principles of space coordinate transformation, investigated arbitrary inclined borehole, derived borehole coordinate components transformation and stress components transformation between borehole coordinate system and in-situ stress coordinate system, and the main conclusions are as follows: (1) Got any inclination of the wellbore coordinate transformation coefficient matrix [L]; (2) Got the transformation equation between the stress components and in-situ stress components; (3) Through research and analysis the coordinate components transformation and the stress components transformation applicated in the engineering fields, aware of their applicated in engineering fields mainly to solve the basic problems of the mechanics and the kinematics.
\end{abstract}

Keywords- wellbore stability; coordinate transformation; stress components transformation; directional wells; in-situ stress

\section{INTRODUCTION}

During mechanical analysis of wellbore stability in Directional Well, we need to build a mechanical model of the borehole wall stress distribution, while establishment of the mechanical model need to convert the three main places stress of the formation to the wellbore axis coordinates stress. Currently, scholars still have no introduce detaily the process of coordinates changes in many monographs and papers about wellbore stability. Therefore, this paper will be mainly a detailed derivation of the transformation process of the coordinate transformation and stress components.

This work was supported by a grant from the National High Technology Research and Development Program of China (863 Program) (Grant No. 2007AA090801-03 and 2007AA09A103-03), the Basic Research Subject of State Key Lab. of Oil \& Gas Reservoir Geology and Exploitation (Southwest 742 Petroleum University) (Grant No G3-1), the Technology Development Subject of Changqing Oilfield Company (Grant No 12ZJ-KF-001).

\section{THE BASIC PRINCIPLES OF COORDINATE AND STRESS TRANSFORMTIONG}

\section{A. CauChy formula (oblique section stress formula)}

If $O$ is any point on the stress object, we know a group of six independent stress components $\sigma_{x}, \sigma_{y}, \sigma_{z}, \tau_{x y}, \tau_{y z}, \tau_{z x}$. In order to seek the stress on any oblique section outside the normal $\mathbf{n}$ through the $O$ point, we truncate a small tetrahedral units from the A point, and establish the coordinate system $(x, y, z)$, its base vector $\left\{\mathbf{e}_{x}, \mathbf{e}_{y}, \mathbf{e}_{z}\right\}$ as shown in Fig.1.

Assume that regardless of the tetrahedron OABC physical, the direction cosine of outward normal $\mathbf{n}$ of oblique section were recorded as:

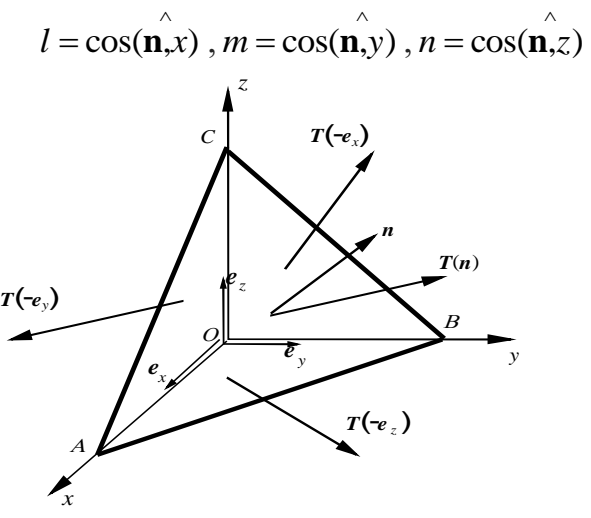


If the area of oblique section $A B C$ is $d S$, the distance from point $O$ to the $A B C$ is $d h$, volume force is $\mathbf{F}$. So the area of three oblique section $O B C, O C A, O A B$ is $l d S, m d S, n d S$ and the volume of tetrahedron $O A B C$ is $d h d S / 3$, thus according to tetrahedral equilibrium conditions derived:

$\mathbf{T}(\mathbf{n}) d S+\mathbf{T}\left(-\mathbf{e}_{x}\right) l d S+\mathbf{T}\left(-\mathbf{e}_{y}\right) m d S+\mathbf{T}\left(-\mathbf{e}_{z}\right) n d S+\mathbf{F} d h d S / 3=0$

Because of $\mathbf{T}(-\mathbf{n})=-\mathbf{T}(\mathbf{n})$, as the volume force $\mathbf{F} d h d S / 3$ is a smaller amount of high-end than the surface force, so ignored the volume force available:

$$
\mathbf{T}(\mathbf{n})=\mathbf{T}\left(\mathbf{e}_{x}\right) l+\mathbf{T}\left(\mathbf{e}_{y}\right) m+\mathbf{T}\left(\mathbf{e}_{z}\right) n
$$

This is the famous CauChy formula, also known as oblique section stress formula $\mathbf{T}(\mathbf{n})$, its essence is equilibrium conditions of a tiny tetrahedral ${ }^{[1]}$.

Making slant stress vector $\mathbf{T}(\mathbf{n})$ along the axis direction decompose that get:

$$
\mathbf{T}(\mathbf{n})=T_{x} \mathbf{e}_{x}+T_{y} \mathbf{e}_{y}+T_{z} \mathbf{e}_{z}
$$

While the three stress component vectors (a total of nine component) under cartesian coordinate system is:

$$
\left\{\begin{array}{l}
\mathbf{T}\left(\mathbf{e}_{x}\right)=\sigma_{x x} \mathbf{e}_{x}+\tau_{y x} \mathbf{e}_{y}+\tau_{z x} \mathbf{e}_{z} \\
\mathbf{T}\left(\mathbf{e}_{y}\right)=\tau_{x y} \mathbf{e}_{x}+\sigma_{y y} \mathbf{e}_{y}+\tau_{z y} \mathbf{e}_{z} \\
\mathbf{T}\left(\mathbf{e}_{z}\right)=\tau_{x z} \mathbf{e}_{x}+\tau_{y z} \mathbf{e}_{y}+\sigma_{z z} \mathbf{e}_{z}
\end{array}\right.
$$

Thus, we can get the component form of oblique section formula from Eq. (4) and Eq. (5):

$$
\left\{\begin{array}{l}
T_{x}=\sigma_{x x} l+\tau_{y x} m+\tau_{z x} n \\
T_{y}=\tau_{x y} l+\sigma_{y y} m+\tau_{z y} n \\
T_{z}=\tau_{x z} l+\tau_{y z} m+\sigma_{z z} n
\end{array}\right.
$$

The matrix form of Eq. (6) is:

$$
\left[\begin{array}{c}
T_{x} \\
T_{y} \\
T_{z}
\end{array}\right]=\left[\begin{array}{ccc}
\sigma_{x x} & \tau_{x y} & \tau_{x z} \\
\tau_{y x} & \sigma_{y y} & \tau_{y z} \\
\tau_{z x} & \tau_{z y} & \sigma_{z z}
\end{array}\right]\left[\begin{array}{c}
l \\
m \\
n
\end{array}\right]
$$

At this point, the normal stress on the oblique section can write that:

$$
\begin{aligned}
\sigma_{n} & =\mathbf{T}(\mathbf{n}) \cdot \mathbf{n}=T_{x} l+T_{y} m+T_{z} n \\
& =\sigma_{x x} l^{2}+\sigma_{y y} m^{2}+\sigma_{z z} n^{2}+\tau_{x y} l m+\tau_{y z} m n+\tau_{z x} n l
\end{aligned}
$$

Tangential shear stress can write that:

$$
\tau_{n}=\sqrt{\|\mathbf{T}(\mathbf{n})\|^{2}-\sigma_{n}^{2}},\|\mathbf{T}(\mathbf{n})\|=\sqrt{T_{x}^{2}+T_{y}^{2}+T_{z}^{2}}
$$

\section{B. The basic principles of coordinate transformation}

If $(x, y, z)$ is a rectangular coordinate, $\left(x^{\prime}, y^{\prime}, z^{\prime}\right)$ is a new coordinates which come form rotating $(x, y, z)$ coordinate system. So the original coordinate system is $(x, y, z)$, the new coordinate system is $\left(x^{\prime}, y^{\prime}, z^{\prime}\right)$, and the unit base vector of the original coordinate system $\left(x^{\prime}, y^{\prime}, z^{\prime}\right)$ is $\left\{\mathbf{e}_{x}, \mathbf{e}_{y}, \mathbf{e}_{z}\right\}$, the new is $\left\{\mathbf{e}_{x}^{\prime}, \mathbf{e}_{y}^{\prime}, \mathbf{e}_{z}^{\prime}\right\}$.

If the projection(three direction cosine) of $\mathbf{e}_{x}^{\prime}$ in the old coordinates of each axis is $l_{1}, m_{1}, n_{1}$, the projection of $\mathbf{e}_{y}^{\prime}$ is $l_{2}, m_{2}, n_{2}$, and the projection of $\mathbf{e}_{z}^{\prime}$ is $l_{3}, m_{3}, n_{3}$. The unit base vector of new and old coordinate system has the following relations $^{[1,2]}$ :

$$
\left[\begin{array}{l}
\boldsymbol{e}_{x}^{\prime} \\
\boldsymbol{e}_{y}^{\prime} \\
\boldsymbol{e}_{z}^{\prime}
\end{array}\right]=\left[\begin{array}{lll}
l_{1} & m_{1} & n_{1} \\
l_{2} & m_{2} & n_{2} \\
l_{3} & m_{3} & n_{3}
\end{array}\right]\left[\begin{array}{l}
\boldsymbol{e}_{x} \\
\boldsymbol{e}_{y} \\
\boldsymbol{e}_{z}
\end{array}\right]
$$

In Eq. (10), the matrix compose of $l_{1}, m_{1}, n_{1}, l_{2}, m_{2}, n_{2}$ and $l_{3}, m_{3}, n_{3}$ is coordinate transformation matrix.

Seen the three planes $(O x y, O y z, O x z)$ in the new coordinate system as the slope in the original coordinate, then we can derive the relationship of the stress components between the original and the new coordinate system by using the CauChy foumula, in the following article coordinate transform and stress component transform will be derived according to the above transformation principles.

\section{DERIVATION OF THE COORDINATE AND STRESS COMPONENT TRANSFOMATION OF DEVIATED BOREHOLE}

Select a coordinate system which has the same direction with the main ground stress $\sigma_{H}, \sigma_{h}, \sigma_{v}$ (Fig.2). For convenience, we establish a cartesian coordinate system $(x, y, z)$, of which the $O z$ axis corresponds the the well shaft, $O x$ and $O y$ located in the plane perpendicular to well axis ${ }^{[3,4,5]}$.

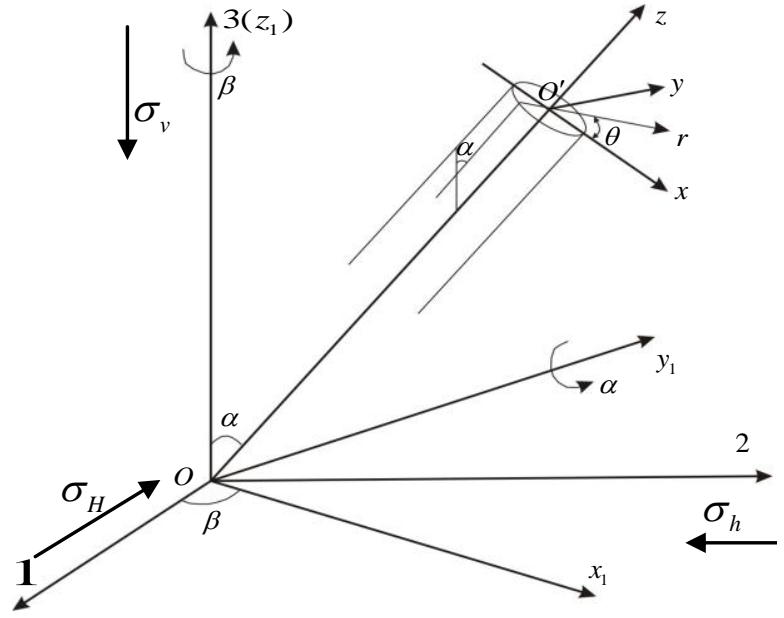

Figure 2. Schematic diagram of coordinate transformation

\section{A. The derivation of coordinate transformation}

In order to establish the converse relationship between the coordinates $(x, y, z)$ and the coordinates $(1,2,3)$, We need to introduce the intermediate coordinates system $\left(x_{1}, y_{1}, z_{1}\right)$, assuming that the unit base vector of coordinate system $(1,2,3)$ is $\left\{\mathbf{e}_{1}, \mathbf{e}_{2}, \mathbf{e}_{3}\right\}$, the unit base vector of intermediate coordinate system $\left(x_{1}, y_{1}, z_{1}\right)$ is $\left\{\mathbf{e}_{x 1}, \mathbf{e}_{y 2}, \mathbf{e}_{z 3}\right\}$, the unit base vector of coordintae system $(x, y, z)$ is $\left\{\mathbf{e}_{x}, \mathbf{e}_{y}, \mathbf{e}_{z}\right\}$. Now rotate the coordinate $(1,2,3)$ in the following manner (Fig.2): 
- Firstly, according to the right-hand rule, coordinate $(1,2,3)$, rotate angle $\beta$ based on coordinate axis 3 , we can get the coordinate system of $\left(x_{1}, y_{1}, z_{1}\right)$. The rotate angle $\beta$ should be the included angle of hole deviation azimuth and the maximum principle stress (the drift azimuth), the hole deviation azimuth is the included angle between north direction and the projection traces of directional well axis in the horizontal plane. The orientation of maximum horizontal principle stress is the included angle between the stress direction and the north. After rotation, the projection of the intermediate coordinate system $\left(x_{1}, y_{1}, z_{1}\right)$ of unit base $\mathbf{e}_{x 1}$ in the coordinate $(1,2,3)$ axis of coordinate is $l_{1}^{\prime}=\cos \beta, m_{1}^{\prime}=\sin \beta, n_{1}^{\prime}=0$, the projection of $\mathbf{e}_{y 1}$ in each coordinate axis is $l_{2}^{\prime}=-\sin \beta, \quad m_{2}^{\prime}=\cos \beta, \quad n_{2}^{\prime}=0$, the projection of $\mathbf{e}_{21}$ in each coordinate axis is $l_{3}^{\prime}=0, m_{3}^{\prime}=0, n_{3}^{\prime}=1$, so the unit base vector of two coordinate system have the following relationship:

$$
\left[\begin{array}{l}
\mathbf{e}_{x 1} \\
\mathbf{e}_{y 1} \\
\mathbf{e}_{z 1}
\end{array}\right]=\left[\begin{array}{ccc}
\cos \beta & \sin \beta & 0 \\
-\sin \beta & \cos \beta & 0 \\
0 & 0 & 1
\end{array}\right]\left[\begin{array}{l}
\mathbf{e}_{1} \\
\mathbf{e}_{2} \\
\mathbf{e}_{3}
\end{array}\right]
$$

- Secondly, according to the right-hand principle rotate angle $\alpha$ based on coordinate axis $y_{1}$, we can get the coordinate system $(x, y, z) . \alpha$ is the hole deviation angle, which means the included angle between the borehole axis of directional well and the vertical line. After totation, the projection of the unit base vector $\mathbf{e}_{x}$ of coordinate system $(x, y, z)$ in the coordinate axis of coordinate system $\left(x_{1}, y_{1}, z_{1}\right)$ is $l_{1}^{\prime \prime}=\cos \alpha, m_{1}^{\prime \prime}=0, n_{1}^{\prime \prime}=$ $-\sin \alpha$. The projection of $\mathbf{e}_{y}$ in each coordinate axis is $l_{2}^{\prime \prime}=0$, $m_{2}^{\prime \prime}=1, n_{2}^{\prime \prime}=0$, the projection of $\mathbf{e}_{z}$ in each coordinate axis is $l_{3}^{\prime \prime}=\sin \alpha, m_{3}^{\prime \prime}=0, n_{3}^{\prime \prime}=\cos \alpha$, so the unit basis vectors of two coordinate system have the following relationship:

$$
\left[\begin{array}{l}
\mathbf{e}_{x} \\
\mathbf{e}_{y} \\
\mathbf{e}_{z}
\end{array}\right]=\left[\begin{array}{ccc}
\cos \alpha & 0 & -\sin \alpha \\
0 & 1 & 0 \\
\sin \alpha & 0 & \cos \alpha
\end{array}\right]\left[\begin{array}{l}
\mathbf{e}_{x 1} \\
\mathbf{e}_{y 1} \\
\mathbf{e}_{z 1}
\end{array}\right]
$$

Combining Eq. (11) and Eq. (12) can be obtained:

$$
\left[\begin{array}{l}
\mathbf{e}_{x} \\
\mathbf{e}_{y} \\
\mathbf{e}_{z}
\end{array}\right]=\left[\begin{array}{lll}
l_{1} & m_{1} & n_{1} \\
l_{2} & m_{2} & n_{2} \\
l_{3} & m_{3} & n_{3}
\end{array}\right]\left[\begin{array}{l}
\mathbf{e}_{1} \\
\mathbf{e}_{2} \\
\mathbf{e}_{3}
\end{array}\right]=\left[\begin{array}{ccc}
\cos \alpha \cos \beta & \cos \alpha \sin \beta & -\sin \alpha \\
-\sin \beta & \cos \beta & 0 \\
\sin \alpha \cos \beta & \sin \alpha \sin \beta & \cos \alpha
\end{array}\right]\left[\begin{array}{l}
\mathbf{e}_{1} \\
\mathbf{e}_{2} \\
\mathbf{e}_{3}
\end{array}\right]
$$

So we can get the coordinate transformation coefficients of deviate well borehole:

$$
[L]=\left[\begin{array}{lll}
l_{1} & m_{1} & n_{1} \\
l_{2} & m_{2} & n_{2} \\
l_{3} & m_{3} & n_{3}
\end{array}\right]=\left[\begin{array}{ccc}
\cos \alpha \cos \beta & \cos \alpha \sin \beta & -\sin \alpha \\
-\sin \beta & \cos \beta & 0 \\
\sin \alpha \cos \beta & \sin \alpha \sin \beta & \cos \alpha
\end{array}\right]
$$

\section{B. The derivation of stress component transformtion}

We can get the far-field stress components of borehole coordinate $\sigma_{x x}, \sigma_{y y}, \sigma_{z z}, \tau_{x y}, \tau_{y z}, \tau_{z x}{ }^{[3,4,5]}$ as Fig.3 has shown by rotating the main stress component from coordinate $(1,2,3)$ to coordinate $(x, y, z)$, which is shown in Fig.2. When solving the problem, we can see $y O z, x O z, x O y$ three faces as the inclined surface of coordinate $(1,2,3)$, then we can deduce the transformation relationship of stress component in the new coordinate system.

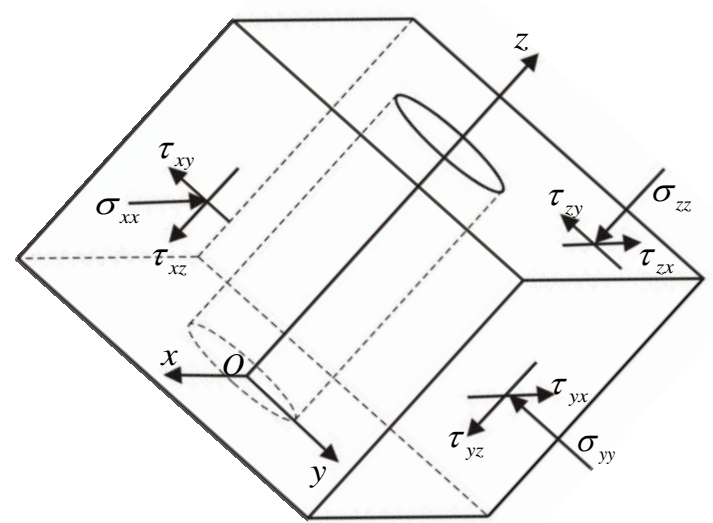

Figure 3. The stress components of wellbore coordinates

\section{1) For plane $y \mathrm{Oz}$}

In the coordinate of the borehole, for the assuming $y \mathrm{Oz}$ plane, the outside normal direction unit basis vector $\mathbf{e}_{x}$, the insitu stress components in coordinate $(1,2,3)$ is $\sigma_{H}, \sigma_{h}, \sigma_{v}$, the projection of unit basis vector $\mathbf{e}_{x}$ in coordinate system $(1,2,3)$ is $l_{1}, m_{1}, n_{1}$ (values in Eq.(14)), since the unit basis vector of coordinate system $(1,2,3)$ is $\left\{\mathbf{e}_{1}, \mathbf{e}_{2}, \mathbf{e}_{3}\right\}$, so we can decompose the stress vector $\mathbf{T}\left(\mathbf{e}_{x}\right)$ in this cross-section, which is in the coordinate $(1,2,3)$ :

$$
T\left(\mathbf{e}_{x}\right)=T_{x}^{\prime} \mathbf{e}_{1}+T_{y}^{\prime} \mathbf{e}_{2}+T_{z}^{\prime} \mathbf{e}_{3}
$$

By the application of ChauChy formula, the three components of stress vector $\mathbf{T}\left(\mathbf{e}_{x}\right)$ in $y O z$ plane, which is in the si-tu stress coordinate $(1,2,3)$

$$
\left[\begin{array}{c}
T_{x}^{\prime} \\
T_{y}^{\prime} \\
T_{z}^{\prime}
\end{array}\right]=\left[\begin{array}{ccc}
\sigma_{H} & 0 & 0 \\
0 & \sigma_{h} & 0 \\
0 & 0 & \sigma_{v}
\end{array}\right]\left[\begin{array}{l}
l_{1} \\
m_{1} \\
n_{1}
\end{array}\right]
$$

$\mathbf{T}\left(\mathbf{e}_{x}\right)$ has the normal stress component $\sigma_{x x}$ and shear stress component $\tau_{x y}, \tau_{y z}$ in the cross section of new coordinate and the normal line of the new coordinate is $\mathbf{e}_{x}$ :

$$
\left\{\begin{array}{l}
\sigma_{x x}=\mathbf{T}\left(\mathbf{e}_{x}\right) \cdot \mathbf{e}_{x}=\left(T_{x}^{\prime} \mathbf{e}_{1}+T_{y}^{\prime} \mathbf{e}_{2}+T_{z}^{\prime} \mathbf{e}_{3}\right) \cdot \mathbf{e}_{x}=\mathrm{T}_{x}^{\prime} l_{1}+\mathrm{T}_{y}^{\prime} m_{1}+\mathrm{T}_{z}^{\prime} n_{1} \\
\tau_{x y}=\mathbf{T}\left(\mathbf{e}_{x}\right) \cdot \mathbf{e}_{y}=\left(T_{x}^{\prime} \mathbf{e}_{1}+T_{y}^{\prime} \mathbf{e}_{2}+T_{z}^{\prime} \mathbf{e}_{3}\right) \cdot \mathbf{e}_{y}=\mathrm{T}_{x}^{\prime} l_{2}+\mathrm{T}_{y}^{\prime} m_{2}+\mathrm{T}_{z}^{\prime} n_{2} \\
\tau_{x z}=\mathbf{T}\left(\mathbf{e}_{x}\right) \cdot \mathbf{e}_{z}=\left(T_{x}^{\prime} \mathbf{e}_{1}+T_{y}^{\prime} \mathbf{e}_{2}+T_{z}^{\prime} \mathbf{e}_{3}\right) \cdot \mathbf{e}_{z}=\mathrm{T}_{x}^{\prime} l_{3}+\mathrm{T}_{y}^{\prime} m_{3}+\mathrm{T}_{z}^{\prime} n_{3}
\end{array}\right.
$$

The Eq. (17) can be explained with matrix:

$$
\left[\begin{array}{l}
\sigma_{x x} \\
\tau_{x y} \\
\tau_{x z}
\end{array}\right]=\left[\begin{array}{lll}
l_{1} & m_{1} & n_{1} \\
l_{2} & m_{2} & n_{2} \\
l_{3} & m_{3} & n_{3}
\end{array}\right]\left[\begin{array}{c}
\mathrm{T}_{x}^{\prime} \\
\mathrm{T}_{y}^{\prime} \\
\mathrm{T}_{z}^{\prime}
\end{array}\right]=[L]\left[\begin{array}{c}
\mathrm{T}_{x}^{\prime} \\
\mathrm{T}_{y}^{\prime} \\
\mathrm{T}_{z}^{\prime}
\end{array}\right]
$$

So we can get the conclusion from Eq. (16) and Eq. (18):

$$
\begin{aligned}
\left(\begin{array}{lll}
\sigma_{x x} & \tau_{x y} & \tau_{x z}
\end{array}\right) & =\left[\begin{array}{c}
\mathrm{T}_{x}^{\prime} \\
\mathrm{T}_{y}^{\prime} \\
\mathrm{T}_{z}^{\prime}
\end{array}\right]^{T}[L]^{T}=\left[\begin{array}{c}
l_{1} \\
m_{1} \\
n_{1}
\end{array}\right]^{T}\left[\begin{array}{ccc}
\sigma_{H} & 0 & 0 \\
0 & \sigma_{h} & 0 \\
0 & 0 & \sigma_{v}
\end{array}\right]^{T}[L]^{T} \\
& =\left(\begin{array}{lll}
l_{1} & m_{1} & n_{1}
\end{array}\right)\left[\sigma_{H h v}\right][L]^{T}
\end{aligned}
$$

In the same way, we can deduce to plane $x O y$ and $x O z$. 


\section{2) For plane $x \mathrm{Oz}$}

Under the coordinate of wellbore, for the assuming plane $x O z$, the outer normal unit basis vector is $\mathbf{e}_{y}$, with the applying of CauChy formula, we can get the normal direct stress component $\sigma_{y y}$ and tangential shear stress component $\tau_{y x}, \tau_{y z}$ of plane $x O z$.

$$
\left(\begin{array}{lll}
\tau_{y x} & \sigma_{y y} & \tau_{y z}
\end{array}\right)=\left(\begin{array}{lll}
l_{2} & m_{2} & n_{2}
\end{array}\right)\left[\sigma_{H h v}\right][L]^{T}
$$

\section{3) For plane $x \mathrm{Oy}$}

Under the coordinate of wellbore, for the assuming surface of $x O y$, the outer normal unit basis vector is $\mathbf{e}_{z}$, with the applying of CauChy formula, we can get the normal direct stress component $\sigma_{z z}$ and tangential shear stress component $\tau_{z x}$, $\tau_{z y}$ of $x O y$ surface.

$$
\left(\begin{array}{lll}
\tau_{z x} & \tau_{z y} & \sigma_{z z}
\end{array}\right)=\left(\begin{array}{lll}
l_{3} & m_{3} & n_{3}
\end{array}\right)\left[\sigma_{H h v}\right][L]^{T}
$$

4) The transformation relationship formula of stress component

We can get the transformtion relationship formula between situ coordinates and the stress component of borehole coordinate when we combine Eq. (19) and Eq. (20):

$$
[\sigma]=\left[\begin{array}{ccc}
\sigma_{x x} & \tau_{x y} & \tau_{x z} \\
\tau_{y x} & \sigma_{y y} & \tau_{y z} \\
\tau_{z x} & \tau_{z y} & \sigma_{z z}
\end{array}\right]=[L]\left[\begin{array}{ccc}
\sigma_{H} & 0 & 0 \\
0 & \sigma_{h} & 0 \\
0 & 0 & \sigma_{v}
\end{array}\right][L]^{T}=[L]\left[\sigma_{H h v}\right][L]^{T}
$$

We can rewrite the Eq. (22) to the following form of stress component based on the theorem that their tangentials are reciprocal equal.

$$
\left\{\begin{array}{l}
\sigma_{x x}=\sigma_{H} \cos ^{2} \alpha \cos ^{2} \beta+\sigma_{h} \cos ^{2} \alpha \sin ^{2} \beta+\sigma_{v} \sin ^{2} \alpha \\
\sigma_{y y}=\sigma_{H} \sin ^{2} \beta+\sigma_{h} \cos ^{2} \beta \\
\sigma_{z z}=\sigma_{H} \sin ^{2} \alpha \cos ^{2} \beta+\sigma_{h} \sin ^{2} \alpha \sin ^{2} \beta+\sigma_{v} \cos ^{2} \alpha \\
\tau_{x y}=-\sigma_{H} \cos \alpha \cos \beta \sin \beta+\sigma_{h} \cos \alpha \cos \beta \sin \beta \\
\tau_{x z}=\sigma_{H} \cos \alpha \sin \alpha \cos ^{2} \beta+\sigma_{h} \cos \alpha \cos \alpha \sin ^{2} \beta+\sigma_{v} \sin \alpha \cos \alpha \\
\tau_{y z}=-\sigma_{H} \sin \alpha \cos \beta \sin \beta+\sigma_{h} \sin \alpha \cos \beta \sin \beta
\end{array}\right.
$$

IV. APPLICATIONS OF COORDINATE AND STRESS COMPONENTS TRANSFORMATION IN OTHER ENGINEERING FIELDS

- Applications in Engineering Mechanics of the oil and gas wells. For example, in the analysis of the directional wellbore stability mechanics. In the design of the research borehole trajectory (especially 3D borehole trajectory) need coordinate conversion, coordinate transformation and stress components transform is also used in research borehole trajectory control.

- Applications in finite element analysis. We often use the local coordinate system of the same direction of the unit, but local coordinate of the units is different, in order to study, we need to take the same coordinate system, the global coordinate system, so if you want apply each component of the unit in the local coordinate system to the global coordinate system, you must need the coordinate conversion ${ }^{[6]}$.
- Applications in mechanical engineering. Such as the space complex surface modeling, spatial kinematic relations, need coordinate transformation ${ }^{[2]}$. It is necessary to examine component changes in the coordinates and vector of points in different coordinate systems, examining mutual sports relations between two coordinate systems, coordinate transformation is frequently used in practical engineering.

- Applications in trajectory analysis. Due to the movement between the various parts of the bodies are often very complex, in order to accurately and precisely describe the law of motion of the bodies, we often employ the global coordinate system and the local coordinate system, a part of the bodies (local coordinate system) under the law of motion used in global coordinate system, you need to first convert it to a global coordinate system.

The main purpose that the coordinate transformation is widely used in these areas is to solve the basic problem on the mechanics, kinematics. Stress component transform mainly solve mechanics transformation relationship in the coordinate system.

\section{CONCLUSIONS}

This paper based on CauChy formula and the basic principles of space coordinate transformation, investigated arbitrary inclined borehole, derived borehole coordinate components transformation and stress components transformation between borehole coordinate system and in-situ stress coordinate system, and the main conclusions are as follows:

- Got any inclination of the wellbore coordinate transformation coefficient matrix [L].

- Got the transformation equation $[\sigma]=[\mathrm{L}]\left[\sigma_{H h v}\right][\mathrm{L}]^{T}$ between the stress components $\left(\sigma_{x x}, \sigma_{y y}, \sigma_{z z}, \tau_{x y}, \tau_{y z}, \tau_{z x}\right)$ and in-situ stress components $\left(\sigma_{H}, \sigma_{h}, \sigma_{v}\right)$.

- Through research and analysis the coordinate components transformation and the stress components transformation applicated in the engineering fields, aware of their applicated in engineering fields mainly to solve the basic problems of the mechanics and the kinematics.

\section{REFERENCES}

[1] CHEN Mingxiang. Elastic-plastic mechanics[M]. Beijing: Science Press, 2007.(in Chinese)

[2] LI Guixian. Geometric modeling and engineering applications of space[M]. Beijing: Higher Education Press, 2007.(in Chinese)

[3] CHEN Mian, JIN Yan, ZHANG Guangqing. Petroleum Engineering Rock Mechanics[M]. Beijing: Science Press, 2008.(in Chinese)

[4] JIN Yan, CHEN Mian, LIU Gonghui, et al. Wellbore Stability Analysis of Extended Reach wells[J]. JOURNAL OF GEOMECHANICS, 1999,5(01).(in Chinese)

[5] MA Tianshou, CHEN Yingjie, QIAO Quanxi, et al. Directional well borehole stability mechanical analysis of Chunxiao Gas Field[J]. WestChina Exploration Engineering, 2009, 22(07). (in Chinese)

[6] ZHU Rongdong. Studying on Mechanical Behavior and Failure Mechanism of Drillstring in Gas Drilling[D]. Chengdu: SouthWest Petroleum University, 2008. (in Chinese) 